\title{
Aligned Magnetic Field, Radiation, and Rotation Effects on Unsteady Hydromagnetic Free Convection Flow Past an Impulsively Moving Vertical Plate in a Porous Medium
}

\author{
Sandeep Naramgari, ${ }^{1}$ Sugunamma Vangala, ${ }^{2}$ and Mohankrishna Penem ${ }^{2}$ \\ ${ }^{1}$ Division of Fluid Dynamics, VIT University, Vellore 632 014, India \\ ${ }^{2}$ Department of Mathematics, Sri Venkateswara University, Tirupati 517 502, India \\ Correspondence should be addressed to Sandeep Naramgari; nsreddy.dr@gmail.com
}

Received 1 July 2014; Accepted 21 September 2014; Published 21 October 2014

Academic Editor: Alberto Cardona

Copyright (C) 2014 Sandeep Naramgari et al. This is an open access article distributed under the Creative Commons Attribution License, which permits unrestricted use, distribution, and reproduction in any medium, provided the original work is properly cited.

\begin{abstract}
We analyse the effects of aligned magnetic field, radiation, and rotation on unsteady hydromagnetic free convection flow of a viscous incompressible electrically conducting fluid past an impulsively moving vertical plate in a porous medium in presence of heat source. An exact solution of the governing equations in dimensionless form is obtained by Laplace transform technique in ramped temperature case. To compare the results obtained in this case with that of isothermal plate, the exact solution of the governing equations is also obtained for isothermal plate and results are discussed graphically in both ramped temperature and isothermal cases.
\end{abstract}

\section{Introduction}

The study of convective heat transfer from a solid body with different geometries embedded in a fluid saturated porous medium has varied and wide applications in many areas of science and engineering such as geothermal reservoirs, drying of porous solids, chemical catalytic reactors, thermal insulators, nuclear waste repositories, heat exchanger devices, enhanced oil and gas recovery, and underground energy transport. An investigation of an influence of magnetic field on viscous incompressible flow of electrically conducting fluid has its importance in many applications such as extrusion of plastics in the manufacture of rayon and nylon, paper industry, and textile industry and in different geophysical cases and so forth.

Keeping the above applications, Krishna et al. [1] studied the effects of thermal radiation and chemical reaction on the steady two-dimensional stagnation point flow of a viscous incompressible electrically conducting fluid over a stretching surface with suction in the presence of heat generation. The combined effects of rotation and radiation on MHD flow past an impulsively started vertical plate with variable temperature were studied by Rajput and Kumar [2]. Sandeep and Sugunamma [3] discussed the effects of inclined magnetic field on unsteady free convection flow of a dusty viscous fluid between two infinite flat plates filled by a porous medium. Jha and Ajibade [4] have studied the unsteady free convective Couette flow of heat generating/absorbing fluid. Saxena and Dubey [5] discussed the unsteady MHD heat and mass transfer free convection flow of polar fluids past a vertical moving porous plate in a porous medium with heat generation and thermal diffusion. The radiation effects on MHD Couette flow with heat transfer between two parallel plates have been examined by Mebine [6]. Vijayalakshmi [7] have studied radiation effects on free convection flow past an impulsively started vertical plate in a rotating fluid. The effect of a uniform transverse magnetic field on the unsteady transient free convection flow of an incompressible viscous electrically conducting fluid between two infinite vertical parallel plates with constant temperature was studied by Rajput and Sahu [8]. Bestman and Adjepong [9] discussed the magnetohydrodynamic free convection flow, with radiative heat transfer, past an infinite moving plate in rotating incompressible, viscous, and optically transparent medium. Sandeep and 
Sugunamma [10] analyzed effects inclined magnetic field on unsteady free convective flow of a dissipative fluid past a vertical plate. Aligned magnetic field and chemical reaction effects on flow past a vertical oscillating plate through porous medium are discussed by Sandeep and Sugunamma [11]. Singh [12] studied hydromagnetic free convection flow past an impulsively started vertical infinite plate in a rotating fluid and he showed that fluid velocity is more influenced by rotation.

The present paper deals with the effects of aligned magnetic field, radiation, and rotation on unsteady hydromagnetic free convection flow of a viscous incompressible electrically conducting fluid past an impulsively moving vertical plate in a porous medium with heat source, under Boussinesq approximation, assuming that the temperature of the plate has a temporarily ramped profile. An exact solution of the governing equations, in dimensionless form, is obtained by Laplace transform technique. To compare the results obtained in this case with that of isothermal plate and exact solution of the governing equations are also obtained for isothermal plate.

\section{Mathematical Formulation}

We consider an unsteady hydromagnetic free convection flow of a viscous incompressible electrically conducting fluid past an impulsively moving infinite vertical plate embedded in a porous medium and consider the coordinate system in such a way that the $x$-axis is taken along the plate in the upward direction, $y$-axis normal to the plane of the plate in the fluid, and $z$-axis perpendicular to $x y$-plane. The fluid is permitted by an aligned magnetic field applied along the direction of $y$-axis at different angles. Both the fluid and plate rotate in unison with a permit uniform angular velocity $\Omega$ about $y$ axis. Initially, at time $t^{*} \leq 0$, both the fluid and plate are at rest and at a constant temperature $T_{\infty}^{*}$. At time $t^{*}>0$, the plate starts moving in $x$ direction with uniform velocity $U_{0}$ and the temperature of the plate is raised or lowered to $T_{\infty}^{*}+\left(T_{w}^{*}-T_{\infty}^{*}\right) t^{*} / t_{0}$ when $t^{*} \leq t_{0}$, and thereafter, for $t^{*}>t_{0}$, it is maintained at the constant temperature $T_{w}^{*}$. The plate is infinite along $x$ and $z$ directions and it is electrically nonconducting with respect to all physical quantities.

Taking into consideration the assumptions made above, the governing equations for laminar free convection flow of a viscous incompressible electrically conducting fluid past a vertical plate in a uniform porous medium with radiative heat transfer, under Boussinesq approximation, in a rotating frame of reference are

$$
\begin{aligned}
& \frac{\partial u^{*}}{\partial t^{*}}+2 \Omega w^{*} \\
& =v \frac{\partial^{2} u^{*}}{\partial y^{2}}+g \beta^{*}\left(T^{*}-T_{\infty}^{*}\right)^{*}-B \operatorname{Sin}^{2} \theta u^{*}-\frac{v}{K_{1}^{*}} u \\
& \frac{\partial w^{*}}{\partial t^{*}}-2 \Omega u^{*}=v \frac{\partial^{2} w^{*}}{\partial y^{2}}-B \operatorname{Cos}^{2} \theta w^{*}-\frac{v}{K_{1}^{*}} w^{*} \\
& \rho c_{p} \frac{\partial T^{*}}{\partial t^{*}}=k \frac{\partial^{2} T^{*}}{\partial y^{2}}-\frac{\partial q_{r}^{*}}{\partial y}+Q_{0}\left(T^{*}-T_{\infty}^{*}\right)
\end{aligned}
$$

where $T^{*}, g, \beta^{*}, v, \sigma, \rho, k, K_{1}^{*}, c_{p}, Q_{0}$, and $q_{r}^{*}$ are, respectively, temperature of the fluid, acceleration due to gravity, volumetric coefficient of thermal expansion, kinematic coefficient of viscosity, electrical conductivity, fluid density, thermal conductivity, permeability of porous medium, specific heat at constant pressure, heat source parameter, and radiative flux vector. Here, $B=\sigma B_{0}^{2} / \rho$ is applied magnetic field.

The initial and boundary conditions are

$$
\begin{gathered}
u^{*}=w^{*}=0, \quad T^{*}=T_{\infty}^{*} \quad \text { for } y \geq 0, t^{*} \leq 0, \\
u^{*}=U_{0}, \quad w^{*}=0 \quad \text { at } y=0 \text { for } t^{*}>0, \\
T^{*}=T_{\infty}^{*}+\left(T_{w}^{*}-T_{\infty}^{*}\right) \frac{t^{*}}{t_{0}} \quad \text { at } y=0 \text { for } 0<t^{*} \leq t_{0}, \\
T^{*}=T_{w}^{*} \quad \text { at } y=0 \text { for } t^{*}>t_{0}, \\
u^{*} \longrightarrow 0, \quad w^{*} \longrightarrow 0, T^{*} \longrightarrow T_{\infty}^{*} \\
\text { as } y \longrightarrow \infty \text { for } t^{*}>0 .
\end{gathered}
$$

We now use Rosseland approximation which leads to the value of radiative heat flux $q_{r}^{*}$ as

$$
q_{r}^{*}=-\frac{4 \sigma^{*}}{3 k^{*}} \frac{\partial T^{* 4}}{\partial y}
$$

where $k^{*}$ is mean absorption coefficient and $\sigma^{*}$ is StefanBoltzmann constant. It may be noted that, by using Rosseland approximation, we limit our analysis to optically thick fluids. Assuming small temperature differences between fluid temperature $T^{*}$ and free stream temperature $T_{\infty}^{*}$, (5) is linearized by expanding $T^{* 4}$ in Taylors series about free stream temperature $T_{\infty}^{*}$, after neglecting second and higher order terms in $\left(T^{*}-T_{\infty}^{*}\right)$; it takes the form

$$
T^{* 4} \cong 4 T_{\infty}^{* 3} T^{*}-3 T_{\infty}^{* 4}
$$

Making use of (5) and (6), (3) becomes

$$
\frac{\partial T^{*}}{\partial t^{*}}=\frac{k}{\rho c_{p}} \frac{\partial^{2} T^{*}}{\partial y^{2}}+\frac{1}{\rho c_{p}} \frac{16 \sigma^{*} T_{\infty}^{* 3}}{3 k^{*}} \frac{\partial^{2} T *}{\partial y^{2}}+\frac{Q_{0}}{\rho c_{p}}\left(T^{*}-T_{\infty}^{*}\right)
$$

Introduce the following nondimensional variables:

$$
\begin{gathered}
Y=\frac{y}{U_{0} t_{0}}, \quad u=\frac{u^{*}}{U_{0}}, \quad w=\frac{w^{*}}{U_{0}}, \quad t=\frac{t^{*}}{t_{0}}, \\
T=\frac{\left(T^{*}-T_{\infty}^{*}\right)}{\left(T_{w}^{*}-T_{\infty}^{*}\right)} .
\end{gathered}
$$


Equations (1), (2), and (3) in the nondimensional form are

$$
\begin{gathered}
\frac{\partial u}{\partial t}+2 K^{2} w=\frac{\partial^{2} u}{\partial Y^{2}}+\operatorname{Gr} T-\left(M_{1}+\frac{1}{K_{1}}\right) u, \\
\frac{\partial w}{\partial t}-2 K^{2} u=\frac{\partial^{2} w}{\partial Y^{2}}-\left(M_{2}+\frac{1}{K_{1}}\right) w, \\
\frac{\partial T}{\partial t}=\frac{(1+R)}{\operatorname{Pr}} \frac{\partial^{2} T}{\partial Y^{2}}+Q T
\end{gathered}
$$

where $K^{2}=\Omega v / U_{0}^{2}$ is rotation parameter, $M^{\prime}=\sigma B_{0}^{2} v / \rho U_{0}^{2}$ is magnetic parameter, $M_{1}=M^{\prime} \operatorname{Sin}^{2} \theta, M_{2}=M^{\prime} \operatorname{Cos}^{2} \theta$ are inclined magnetic field parameters. $K_{1}=K_{1}^{\prime} U_{0}^{2} / v^{2}$ is porosity parameter, $\mathrm{Gr}=g \beta^{\prime} v\left(T_{w}^{\prime}-T_{\infty}^{\prime}\right) / U_{0}^{3}$ is Grashof number, $\mathrm{Pr}=$ $\rho v c_{p} / k$ is Prandtl number, $Q=Q_{0} v / \rho C_{p} U_{0}^{2}$ is the heat source parameter, and $R=16 \sigma^{*} T_{\infty}^{3} / 3 k k^{*}$ is radiation parameter. According to the above nondimensionalization process, the characteristic time $t_{0}$ can be defined as

$$
t_{0}=\frac{v}{U_{0}^{2}}
$$

Making use of (12), the initial and boundary conditions (5), in nondimensional form, reduce to

$$
\begin{gathered}
u=w=0, \quad T=0 \text { for } Y \geq 0, t \leq 0, \\
u=1, \quad w=0 \quad \text { at } Y=0 \text { for } t>0, \\
T=t \quad \text { at } Y=0 \text { for } 0<t \leq 1, \\
T=1 \quad \text { at } Y=0 \text { for } t>1, \\
u \longrightarrow 0, \quad w \longrightarrow 0, \quad T \longrightarrow 0 \text { as } Y \longrightarrow \infty \text { for } t>0 .
\end{gathered}
$$

Combining (9) and (10) in the form of $=u+$, we obtain

$$
\frac{\partial f}{\partial t}-2 i K^{2} f=\frac{\partial^{2} f}{\partial Y^{2}}-M f-\frac{1}{K_{1}} f+\operatorname{Gr} T
$$

where $M=M_{1}+i M_{2}$.

The initial and boundary conditions (13) in combined form are

$$
\begin{gathered}
f=0, \quad T=0 \text { for } Y \geq 0, t \leq 0, \\
f=1 \quad \text { at } Y=0 \text { for } t>0, \\
T=t \quad \text { at } Y=0 \text { for } 0<t \leq 1, \\
T=1 \quad \text { at } Y=0 \text { for } t>1,
\end{gathered}
$$

$$
f \longrightarrow 0, \quad T \longrightarrow 0 \quad \text { as } Y \longrightarrow \infty \text { for } t>0
$$

2.1. Solution of the Problem. It is evident from (11) and (14) that the energy equation (11) is uncoupled from (14). Therefore, first we can obtain the solution for the fluid temperature $T(Y, t)$ by solving (11) and then, using it in (14), the solution for $f(Y, t)$ can be obtained.
Applying Laplace transform technique, (11) and (14) with the help of (15a) reduce to

$$
\begin{gathered}
\frac{d^{2} \tilde{f}}{d Y^{2}}-\left(s+M+\frac{1}{K_{1}}-2 i K^{2}\right) \tilde{f}+\mathrm{Gr} \widetilde{T}=0, \\
\frac{d^{2} \widetilde{T}}{d Y^{2}}-(a+Q) s \widetilde{T}=0,
\end{gathered}
$$

where $a=\operatorname{Pr} /(1+R)$ and $\tilde{f}(Y, s)$ and $\widetilde{T}(Y, s)$ are Laplace transforms of $f(Y, t)$ and $T(Y, t)$, respectively, defined by $\tilde{f}(Y, s)=\int_{0}^{\infty} f(Y, t) e^{-s t} d t$ and $\widetilde{T}(Y, s)=\int_{0}^{\infty} T(Y, t) e^{-s t} d t$, ( $s>0$ being Laplace transform parameter).

The boundary conditions (15b) to (15e) become

$$
\begin{aligned}
& \tilde{f}=\frac{1}{s}, \quad \widetilde{T}=\frac{\left(1-e^{-s}\right)}{s^{2}} \quad \text { at } Y=0, \\
& \tilde{f} \longrightarrow 0, \quad \widetilde{T} \longrightarrow 0 \quad \text { as } Y \longrightarrow \infty .
\end{aligned}
$$

The solutions of (16), subject to the boundary conditions (17), are given by

$$
\begin{gathered}
\tilde{T}(Y, s)=\frac{1-e^{-s}}{s^{2}} e^{-Y \sqrt{(a+Q) s}}, \\
\tilde{f}(Y, s)=\frac{1}{s} e^{-Y \sqrt{s+\lambda}} \\
+\alpha \frac{1-e^{-s}}{s^{2}(s-\beta)}\left\{e^{-Y \sqrt{s+\lambda}}-e^{-Y \sqrt{(a+Q) s}}\right\},
\end{gathered}
$$

where

$$
\begin{gathered}
\lambda=\left(M+\frac{1}{K_{1}}-2 i K^{2}\right), \\
\alpha=\frac{\mathrm{Gr}}{((a+Q)-1)}, \quad \beta=\frac{\lambda}{((a+Q)-1)} .
\end{gathered}
$$

Taking the inverse Laplace transform of (18), the exact solution for the fluid temperature $T(Y, t)$ and fluid velocity $f(Y, t)=u(Y, t)+i w(Y, t)$ is obtained and expressed in the following form as follows.

Here, $f(Y, t)=F(y, t)$ at ramped temperature case

$$
T(Y, t)=P(Y, t)-H(t-1) P(Y, t-1)
$$

$F(Y, t)$

$$
\begin{aligned}
= & \frac{1}{2}\left[e^{Y \sqrt{\lambda}} \operatorname{erfc}\left(\frac{Y}{2 \sqrt{t}}+\sqrt{\lambda t}\right)+e^{-Y \sqrt{\lambda}} \operatorname{erfc}\left(\frac{Y}{2 \sqrt{t}}-\sqrt{\lambda t}\right)\right] \\
& +\alpha\left[F_{1}(Y, t)-H(t-1) F_{1}(Y, t-1)\right],
\end{aligned}
$$


where

$$
\begin{aligned}
& P(Y, t) \\
& =\left(\frac{(a+Q) Y^{2}}{2}+t\right) \operatorname{erfc}\left(\frac{Y \sqrt{(a+Q)}}{2 \sqrt{t}}\right) \\
& -\sqrt{\frac{(a+Q) t}{\pi}} Y e^{-(a+Q) Y^{2} / 4 t}, \\
& F_{1}(Y, t) \\
& =\frac{e^{\beta t}}{2 \beta^{2}}\left\{e^{Y \sqrt{\lambda+\beta}} \operatorname{erfc}\left(\frac{Y}{2 \sqrt{t}}+\sqrt{(\lambda+\beta) t}\right)\right. \\
& \left.+e^{-Y \sqrt{\lambda+\beta}} \operatorname{erfc}\left(\frac{Y}{2 \sqrt{t}}-\sqrt{(\lambda+\beta) t}\right)\right\} \\
& -\left\{\frac{1}{2 \beta^{2}}+\frac{1}{\beta}\left(\frac{t}{2}+\frac{Y}{4 \sqrt{\lambda}}\right)\right\} e^{Y \sqrt{\lambda}} \operatorname{erfc}\left(\frac{Y}{2 \sqrt{t}}+\sqrt{\lambda t}\right) \\
& -\left\{\frac{1}{2 \beta^{2}}+\frac{1}{\beta}\left(\frac{t}{2}-\frac{Y}{4 \sqrt{\lambda}}\right)\right\} \times e^{-Y \sqrt{\lambda}} \operatorname{erfc}\left(\frac{Y}{2 \sqrt{t}}-\sqrt{\lambda t}\right) \\
& -\frac{e^{\beta t}}{2 \beta^{2}}\left\{e^{Y \sqrt{\beta a}} \operatorname{erfc}\left(\frac{Y \sqrt{a+Q}}{2 \sqrt{t}}+\sqrt{\beta t}\right)+e^{-Y \sqrt{\beta(a+Q)}}\right. \\
& \left.\times \operatorname{erfc}\left(\frac{Y \sqrt{(a+Q)}}{2 \sqrt{t}}-\sqrt{\beta t}\right)\right\} \\
& +\frac{1}{\beta^{2}} \operatorname{erfc}\left(\frac{Y \sqrt{(a+Q)}}{2 \sqrt{t}}\right) \\
& +\frac{1}{\beta}\left\{\left(\frac{(a+Q) Y^{2}}{2}+t\right) \operatorname{erfc}\left(\frac{Y \sqrt{(a+Q)}}{2 \sqrt{t}}\right)\right. \\
& \left.-\sqrt{\frac{(a+Q) t}{\pi}} Y e^{-(a+Q) Y^{2} / 4 t}\right\} .
\end{aligned}
$$

In (20) to (22), $\operatorname{erfc}(x)$ is the complementary error function and $H(t-1)$ is the Heaviside unit step function.

2.2. Solution in Case of Isothermal Plate. The analytical solution for the fluid temperature and velocity, represented by (20) and (21), respectively, is obtained for an unsteady hydromagnetic free convection flow of a viscous incompressible electrically conducting fluid near a vertical moving plate with ramped temperature. In order to highlight the effects of ramped temperature distribution within the plate on the fluid flow, it may be worthwhile to compare such a flow with the one near a moving plate with uniform temperature. Taking into account the assumptions made in the present study, the solution for the fluid temperature and velocity for the fluid flow near a vertical moving isothermal plate is obtained and expressed as

$$
T(Y, t)=\operatorname{erfc}\left(\frac{Y}{2} \sqrt{\frac{(a+Q)}{t}}\right),
$$

$$
\begin{aligned}
& f(Y, t) \\
& =\frac{\left(1-\alpha^{\prime}\right)}{2}\left[e^{Y \sqrt{\lambda}} \operatorname{erfc}\left(\frac{Y}{2 \sqrt{t}}+\sqrt{\lambda t}\right)\right. \\
& \left.+e^{-Y \sqrt{\lambda}} \operatorname{erfc}\left(\frac{Y}{2 \sqrt{t}}-\sqrt{\lambda t}\right)\right] \\
& +\frac{\alpha^{\prime} e^{\beta t}}{2}\left[e^{Y \sqrt{\lambda+\beta}} \operatorname{erfc}\left(\frac{Y}{2 \sqrt{t}}+\sqrt{(\lambda+\beta) t}\right)\right. \\
& \left.+e^{-Y \sqrt{\lambda+\beta}} \operatorname{erfc}\left(\frac{Y}{2 \sqrt{t}}-\sqrt{(\lambda+\beta) t}\right)\right] \\
& +\alpha^{\prime} \operatorname{erfc}\left(\frac{Y}{2} \sqrt{\frac{(a+Q)}{t}}\right) \\
& -\frac{\alpha^{\prime} e^{\beta t}}{2}\left[e^{Y \sqrt{\beta(a+Q)}} \operatorname{erfc}\left(\frac{Y}{2} \sqrt{\frac{(a+Q)}{t}}+\sqrt{\beta t}\right)\right. \\
& \left.+e^{-Y \sqrt{\beta(a+Q)}} \operatorname{erfc}\left(\frac{Y}{2} \sqrt{\frac{(a+Q)}{t}}-\sqrt{\beta t}\right)\right],
\end{aligned}
$$

where $\alpha^{\prime}=\alpha / \beta$.

\section{Results and Discussion}

To study the effects of magnetic parameter $M$, rotation parameter $K^{2}$, radiation parameter $R$, porosity parameter $K_{1}$, aligned angle $\theta$, heat source parameter $Q$, and time $t$ taking $\operatorname{Pr}=0.71$ (ionized air) on the flow field the numerical values of fluid velocity and temperature are displayed graphically and discussed. Here aligned angle is taken as $\theta=\pi / 3$ for discussing velocity and temperature profiles and at different aligned angles also the effect on fluid velocity is studied graphically.

From Figure 1, we observed that an increase in the magnetic parameter $M$ leads to a decrease in fluid velocity near ramped temperature as well as in isothermal plates. But initially at isothermal plate velocity takes reverse action. That is, increase in magnetic field causes an increase of velocity; afterwards, velocity decreases gradually by increase in magnetic field. This is due to the fact that the application of an inclined magnetic field to an electrically conducting fluid gives rise to a resistive force which is known as Lorentz force. It is interesting to note that the magnetic field effect is high on fluid velocity near ramped temperature plate compared to isothermal plate.

From Figure 2, it is evident that, for both ramped temperature and isothermal plates, an increase in rotation 


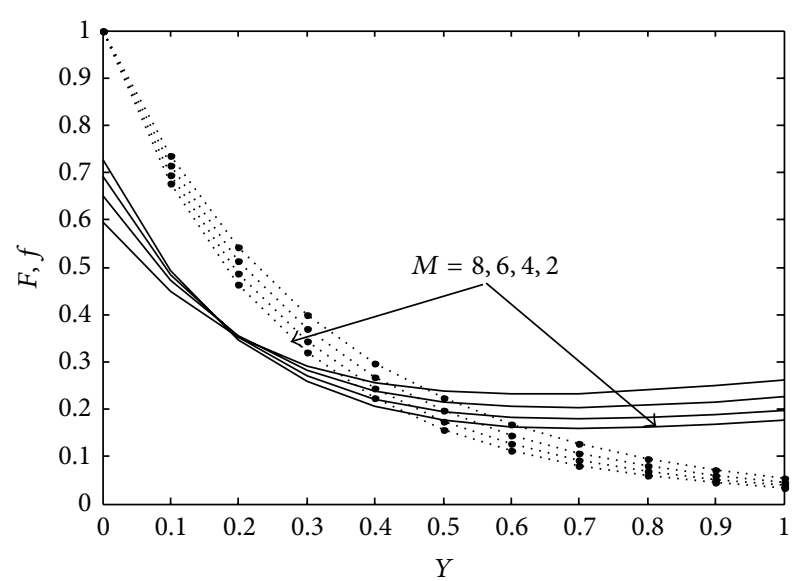

.... Ramped case

— Isothermal case

FIGURE 1: Velocity field for different values of magnetic field parameter $(M)$ when $K^{2}=1, \mathrm{Gr}=5, R=3, K_{1}=0.8, Q=0.4$, and $t=0.4$.

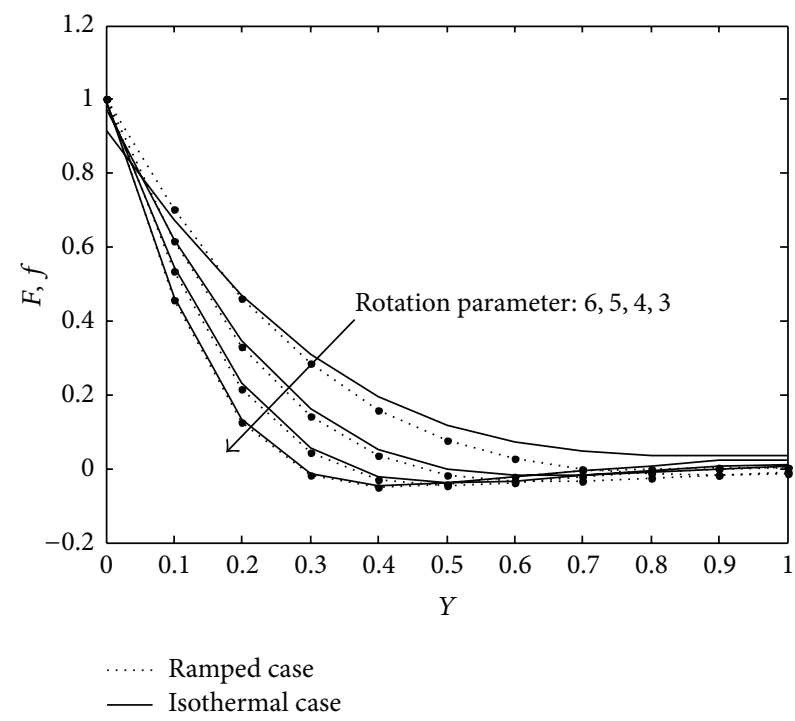

FIGURE 2: Velocity field for different values of rotation parameter $K^{2}$ when $\mathrm{Gr}=5, R=3, K_{1}=1, Q=0.4$, and $t=0.4$.

parameter $K^{2}$ leads to a decrease in the velocity of the fluid near the plate, but there is a slight variation in velocity in ramped temperature case. It may happen due to temperature differences.

Figure 3 depicts the increase in velocity of the fluid near ramped temperature plate by increase of radiation parameter $R$, but it is reversed at isothermal plate. Figure 4 shows the effect of porosity parameter on the fluid velocity at ramped temperature and isothermal plates. It is observed that an increase in porosity parameter causes an increase in fluid velocity. But after certain period it takes reverse direction near isothermal plate.

It is revealed from Figure 5 that the velocity decreases with an increase in the time at isothermal plate, but at ramped temperature, velocity increases by increasing the time $t$.

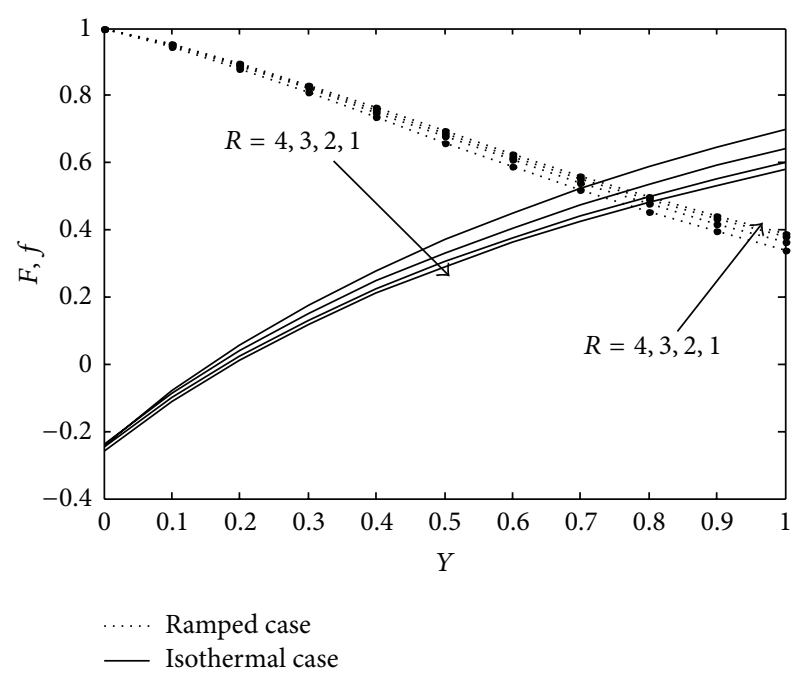

FIGURE 3: Velocity field for different values of radiation parameter $(R)$ when $K^{2}=1, \mathrm{Gr}=5, K_{1}=0.8, Q=0.4$, and $t=0.4$.

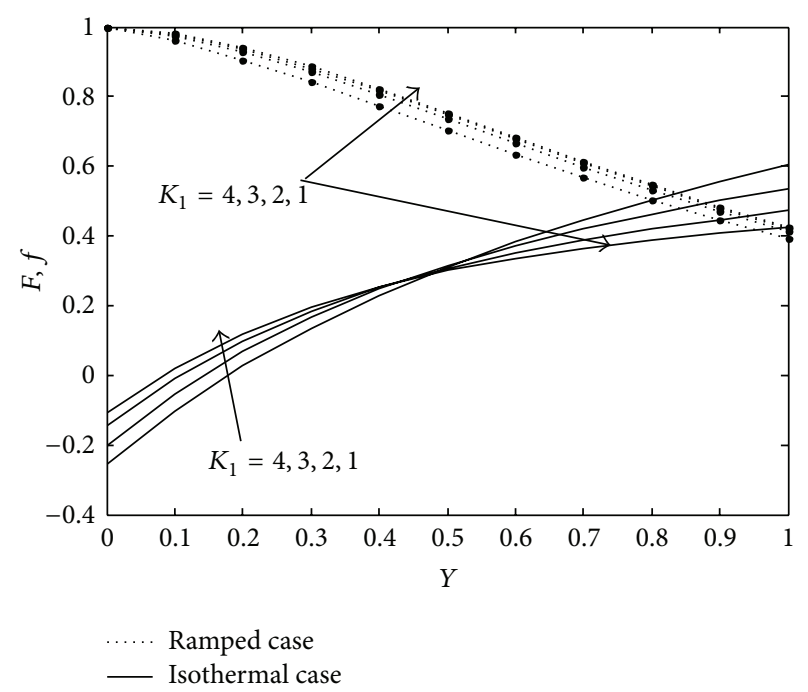

FIGURE 4: Velocity field for different values of porosity parameter $K_{1}$ when $K^{2}=2, R=2, \mathrm{Gr}=2, Q=0.4$, and $t=0.4$.

Figure 6 shows the effect of aligned angle over velocity. From these it is observed that the aligned angle does not show any influence in the velocity of a rotating fluid; here curves happened due to change in magnetic field parameter by changing in aligned angle and this is similar to that case as we discussed in Figure 1. From Figure 7 we observed that increase in heat source parameter causes increase in fluid velocity near ramped temperature plate, but it is reversal near isothermal plate because the heat transfers take reverse action near isothermal plate.

In order to have physical view of fluid temperature, the profiles of fluid temperature are drawn versus boundary layer coordinate $Y$. It is evident from Figure 8 that the fluid temperature $T$ increases by an increase in the radiation parameter at ramped temperature but it is reversed in case 


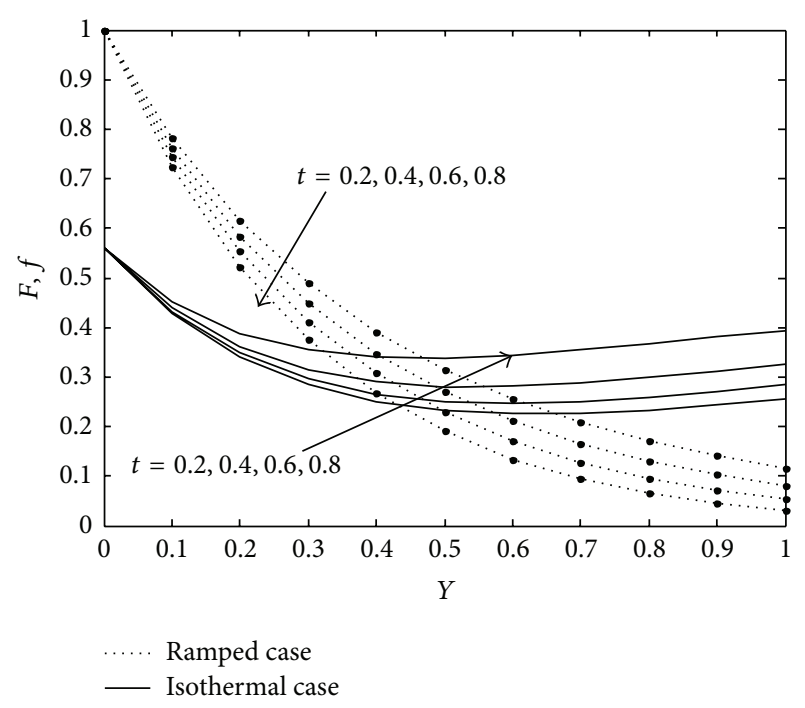

FIGURE 5: Velocity field for different values of time $(t)$ when $K^{2}=4$, $R=1, K_{1}=0.1, Q=0.4$, and $\mathrm{Gr}=5$.

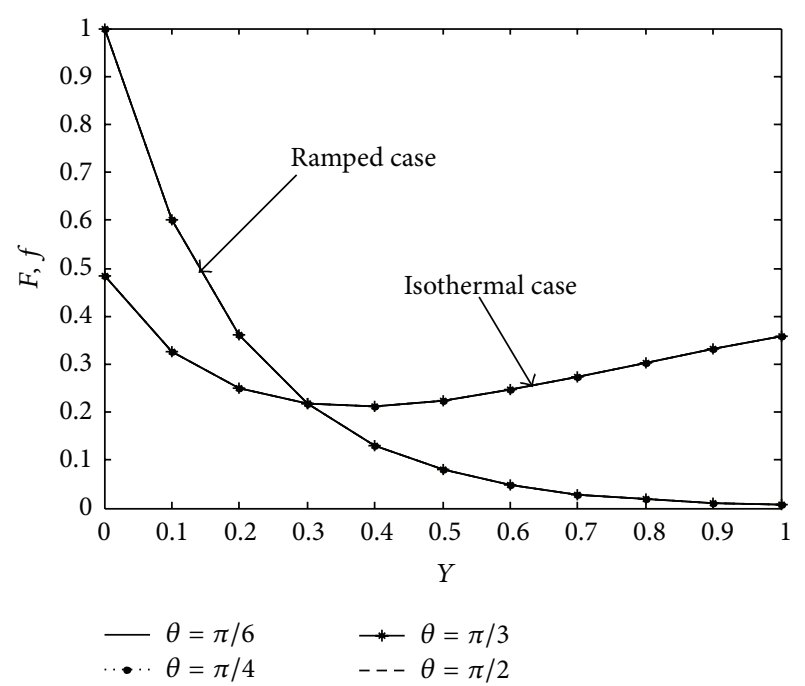

FIGURE 6: Velocity profiles for different values of Aligned angle $\theta$ when $K^{2}=1, R=3, K_{1}=0.1, \mathrm{Gr}=15, Q=0.4$, and $t=0.5$.

of isothermal plate; after certain period it follows isothermal case.

From Figure 9, it is observed that the fluid temperature $T$ increases on increasing Prandtl number Pr at ramped temperature plate and it takes reverse action near isothermal plate. It is evident from Figure 10 that an increase in the heat source parameter increases the fluid temperature for both ramped and isothermal cases.

\section{Conclusions}

The present study investigates the unsteady hydromagnetic free convection boundary layer flow of a viscous incompressible electrically conducting fluid past a ramped temperature impulsively moving plate in a rotating porous medium in

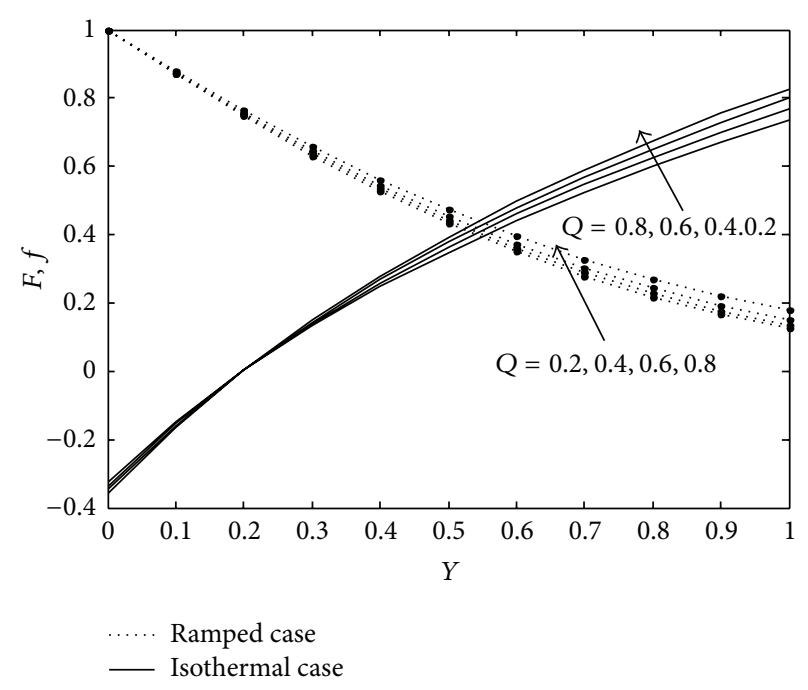

FIGURE 7: Velocity field for different values of heat source parameter $Q$ when $K^{2}=1, M=1, R=3, K_{1}=0.1, \mathrm{Gr}=15$, and $t=1$.

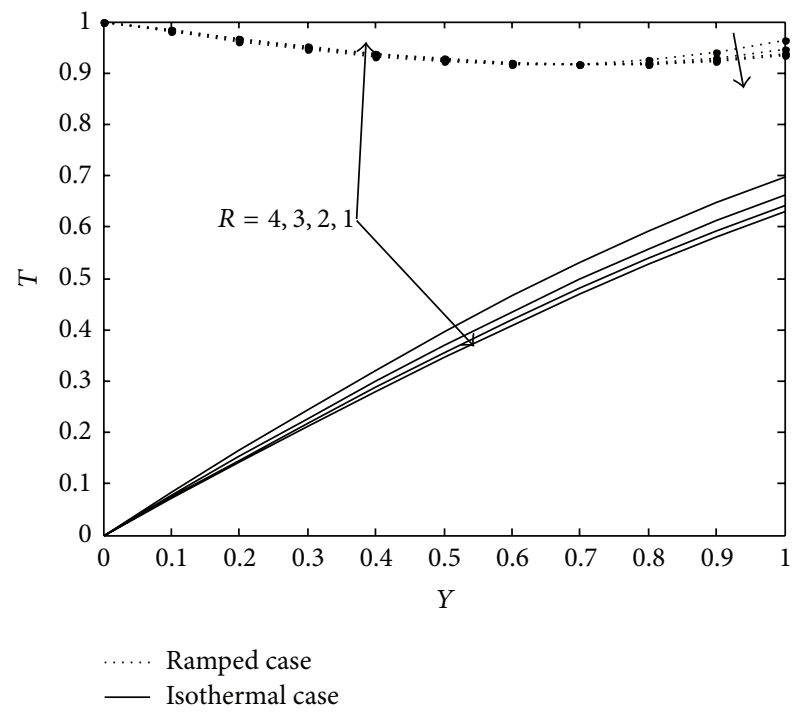

FIgURE 8: Temperature field for different values of $R$ when $K^{2}=1$, $R=2, K_{1}=0.02, \mathrm{Gr}=3, Q=0.4$, and $t=0.5$.

the presence of aligned magnetic field, thermal radiation, and heat source. The significant findings are summarized as follows.

For both ramped temperature and isothermal plates,

(i) magnetic field tends to reduce fluid flow in both the ramped and isothermal cases,

(ii) increase of radiation rotation decreases the fluid flow in the isothermal plate, whereas it increases the fluid flow in the ramped temperature,

(iii) aligned magnetic field angle does not show effect on rotating fluid velocity,

(iv) Prandtl number has tendency to increase the fluid temperature, 


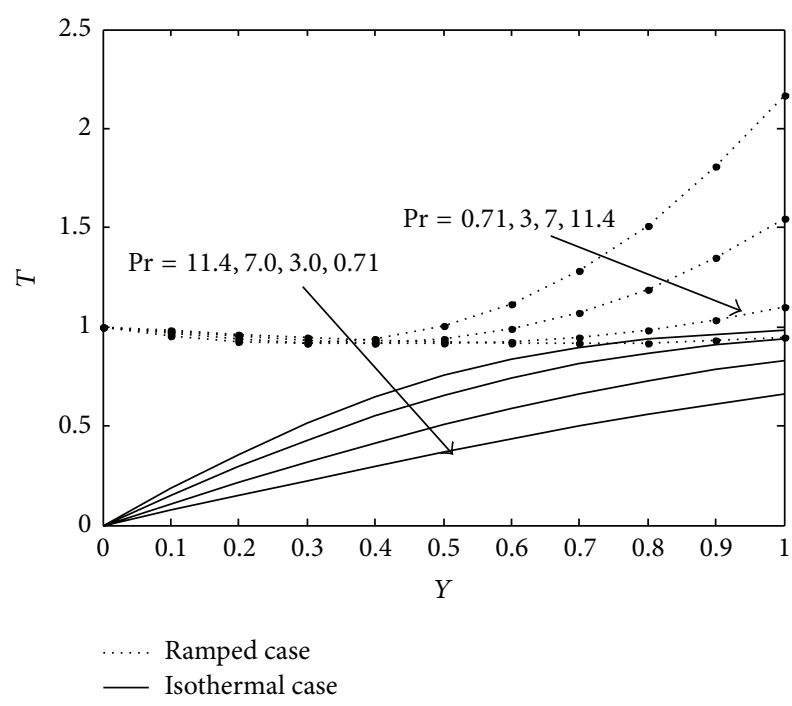

FIgURE 9: Temperature field for different values of Prandtl number (Pr) when $K^{2}=1, R=2, K_{1}=0.02, \mathrm{Gr}=3, Q=0.4$, and $t=0.5$.

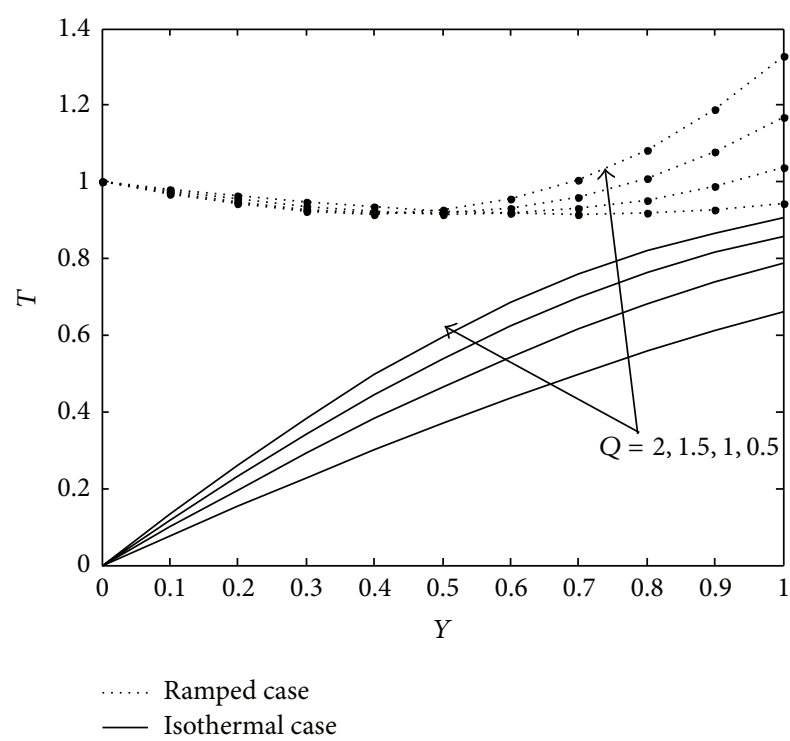

Figure 10: Temperature field for different values of heat source parameter $(Q)$ when $K^{2}=1, R=2, K_{1}=0.2, \mathrm{Gr}=3$, and $t=0.4$.

(v) radiation parameter causes the decrease of temperature in ramped temperature as well as in isothermal case,

(vi) heat source parameter causes the increase in fluid velocity at isothermal case but opposite in ramped case,

(vii) heat source parameter increases the temperature in both cases.

\section{Conflict of Interests}

The authors declare that there is no conflict of interests regarding the publication of this paper.

\section{References}

[1] P. M. Krishna, N. Sandeep, and V. Sugunamma, "Effects of radiation and chemical reaction on MHD convective flow over a permeable stretching surface with suction and heat generation," Walailak Journal of Science and Technology, vol. 11, no. 12, 2014.

[2] U. S. Rajput and S. Kumar, "Combined effects of rotation and radiation on MHD flow past an impulsively started vertical plate with variable temperature," International Journal of Mathematical Analysis, vol. 5, no. 24, pp. 1155-1163, 2011.

[3] N. Sandeep and V. Sugunamma, "Effect of inclined magnetic field on unsteady free convection flow of a dusty viscous fluid between two infinite flat plates filled by a porous medium," International Journal of Applied Mathematics and Modeling, vol. 1, pp. 16-33, 2013.

[4] B. K. Jha and A. O. Ajibade, "Unsteady free convective couette flow of heat generating/absorbing fluid," International Journal of Energy \& Technology, vol. 2, no. 12, pp. 1-9, 2010.

[5] S. S. Saxena and G. K. Dubey, "Unsteady MHD heat and mass transfer free convection flow of polar fluids past a vertical moving porous plate in a porous medium with heat generation and thermal diffusion," Advances in Applied Science Research, vol. 2, no. 4, pp. 259-278, 2011.

[6] P. Mebine, "Radiation effects on MHD Couette flow with heat transfer between two parallel plates," Global Journal of Pure and Applied Mathematics, vol. 3, no. 2, pp. 1-12, 2007.

[7] A. R. Vijayalakshmi, "Radiation effects on free-convection flow past an impulsively started vertical plate in a rotating fluid," Theoretical and Applied Mechanics, vol. 37, no. 2, pp. 79-95, 2010.

[8] U. S. Rajput and P. K. Sahu, "Transient free convection MHD flow between two long vertical parallel plates with constant temperature and variable mass diffusion," International Journal of Mathematical Analysis, vol. 5, no. 33-36, pp. 1665-1671, 2011.

[9] A. R. Bestman and S. K. Adjepong, "Unsteady hydromagnetic free-convection flow with radiative heat transfer in a rotating fluid," Astrophysics and Space Science, vol. 143, no. 1, pp. 73-80, 1988.

[10] N. Sandeep and V. Sugunamma, "Effect of inclined magnetic field on unsteady free convective flow of dissipative fluid past a vertical plate," World Applied Sciences Journal, vol. 22, no. 7, pp. 975-984, 2013.

[11] N. Sandeep and V. Sugunamma, "Aligned magnetic field and chemical reaction effects on flow past a vertical oscillating plate through porous medium," Communications in Applied Sciences, vol. 1, no. 1, pp. 81-105, 2013.

[12] A. K. Singh, "Hydromagnetic free-convection flow past an impulsively started vertical plate in a rotating fluid," International Communications in Heat and Mass Transfer, vol. 11, no. 4, pp. 399-406, 1984. 


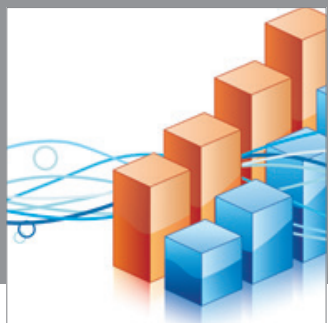

Advances in

Operations Research

mansans

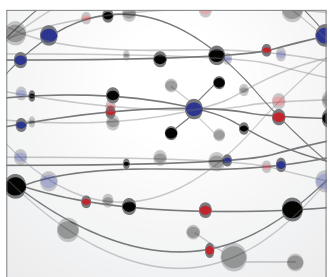

The Scientific World Journal
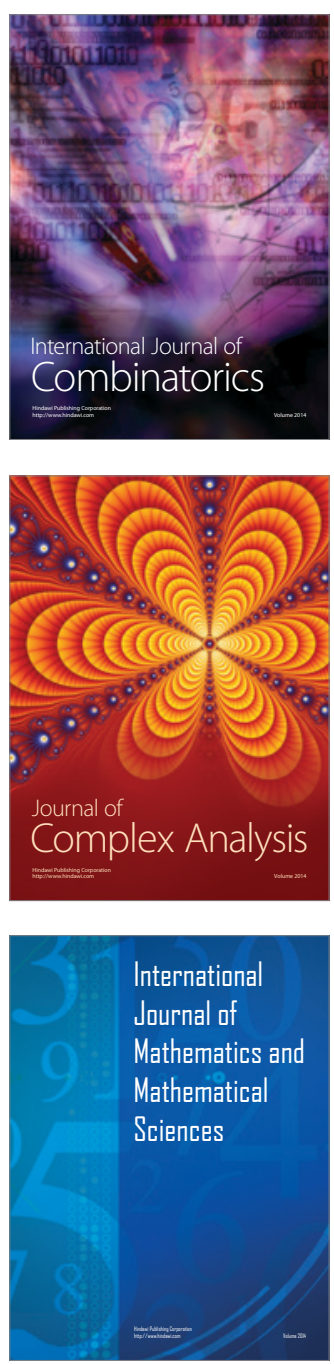
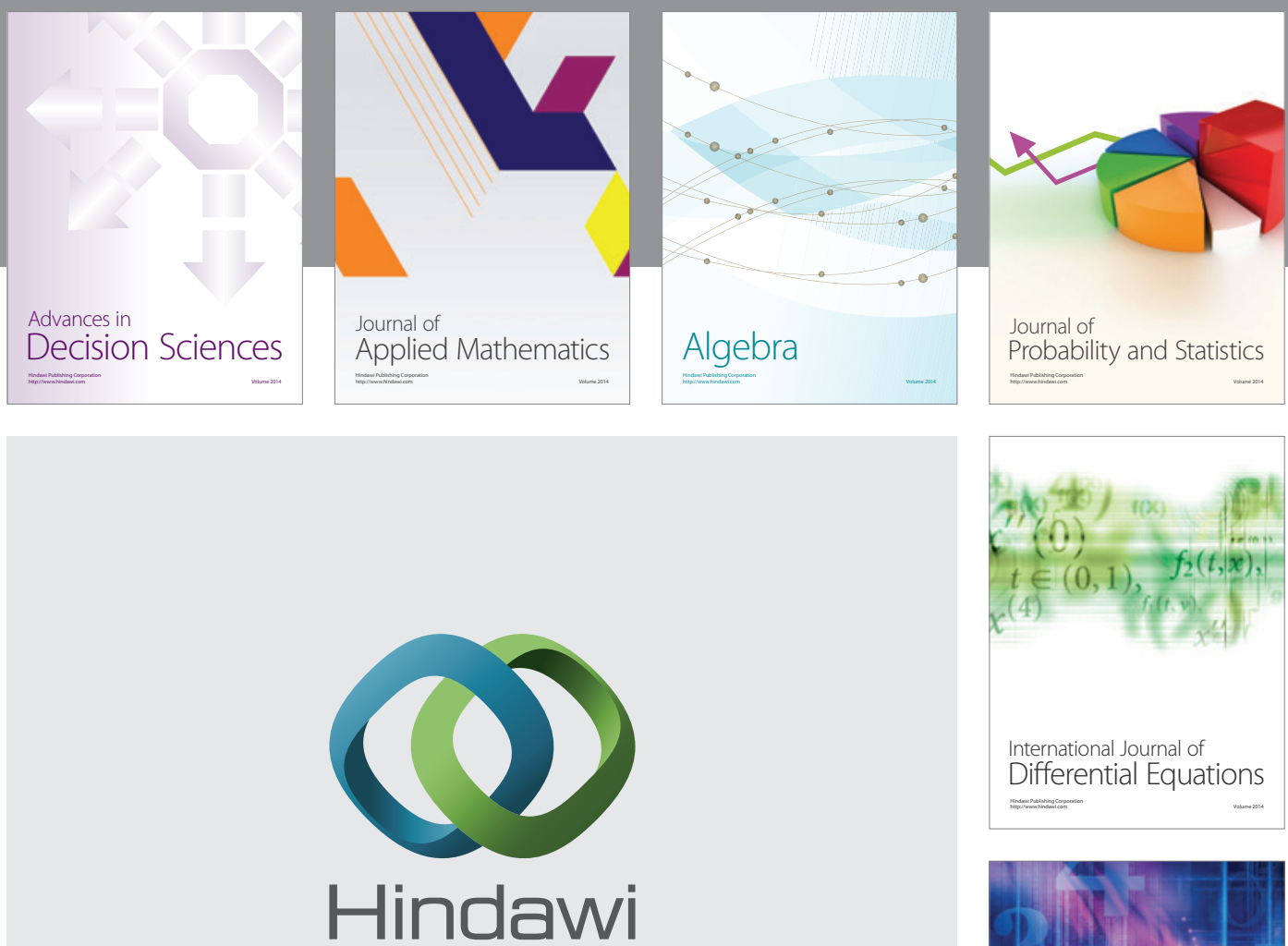

Submit your manuscripts at http://www.hindawi.com
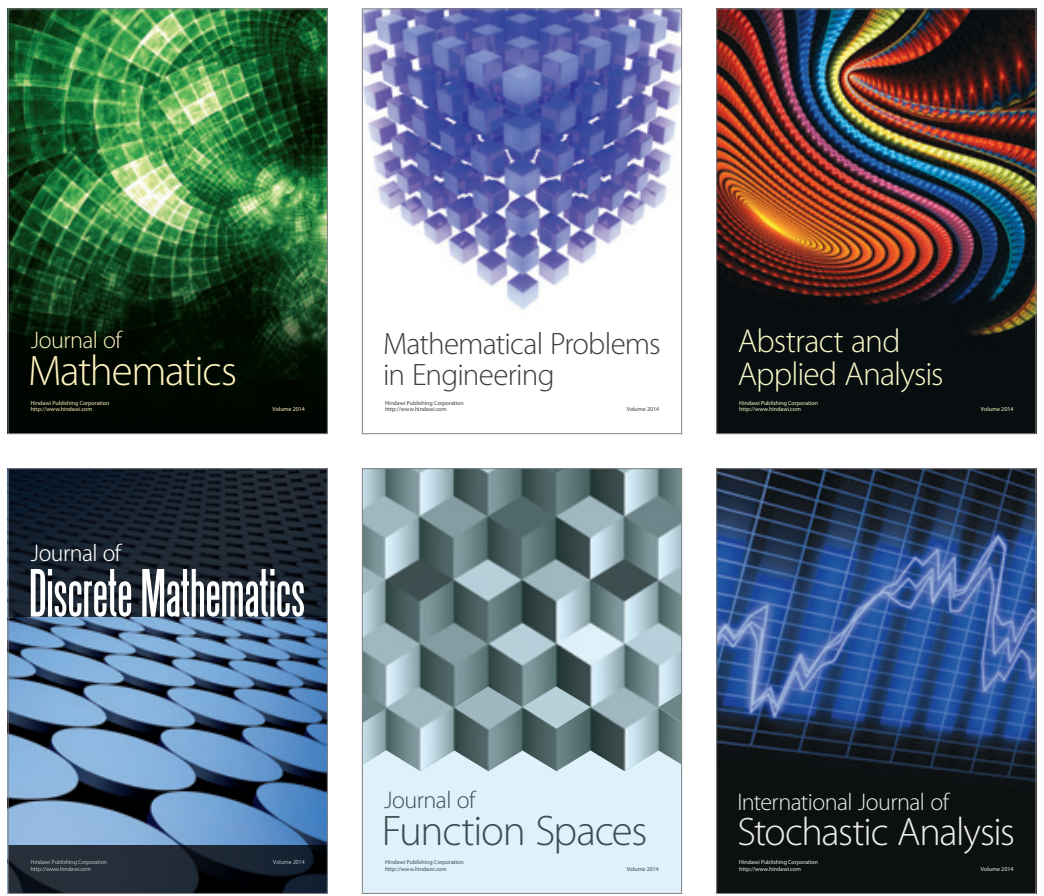

Journal of

Function Spaces

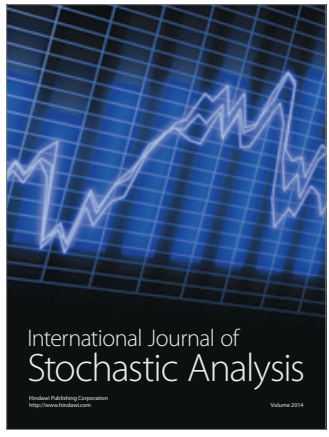

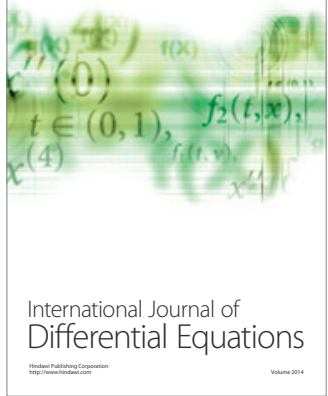
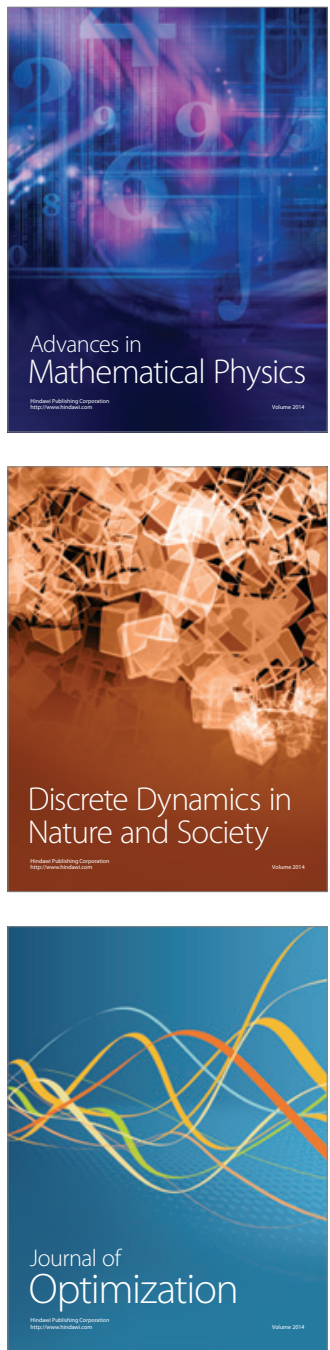